\title{
JPR
}

HEALTH AND HUMAN SCIENCES

\section{Associations Between Language and Gross Motor Skills in Children at Risk for Autism}

\section{Student researcher: Allison Gabouer, Senior}

Infant siblings of children diagnosed with autism spectrum disorder (ASD) are at an elevated risk for ASD and expressive language delays. In previous research, early gross motor scores predicted later language abilities in high-risk (siblings of children with ASD) and low-risk (siblings with no diagnosis) samples. It is unclear if this association is only longitudinal, or if it persists throughout early development. The present study explores concurrently associated gross motor and language development in high- and low-risk samples. Building on previous research, we hypothesized that early gross motor and language skills would be associated in high- and low-risk groups, while controlling for risk status and gender. Infant/toddler language skills were evaluated using the LENA language environment analysis recording device. Gross motor development was assessed using the Mullen Scales of Early Learning. The current sample was selected based on participation in a LENA assessment $(n=19)$ as part of a larger longitudinal study. Infant/toddler ages ranged from 9 to 24 months, with a mean of 18.5 months, and an equal distribution of age and risk status. Partial correlations were used to analyze the association between age-appropriate motor skills and language abilities in both groups. When evaluating outcomes between language and gross motor skills, none of the correlations reached significance $(p<.05)$. In conclusion, the hypothesis was not supported.

This exploratory study did not support a concurrent association between language and motor skills early in development. The lack of significant findings could reflect several factors, including low power (based on small sample size), relatively broad gross motor assessment, or a pure lack of association. Future studies should continue to explore these associations with larger samples and potentially more detailed motor assessments.

Research advisor AJ Schwichtenberg writes: "Allison grew tremendously through this project. She learned that null results are a true part of science and that through them we now know more about how and when early motor skills may relate to language development. Allison incorporated cutting-edge language processing technology to assess early childhood language usage over a full day in a natural language environment."

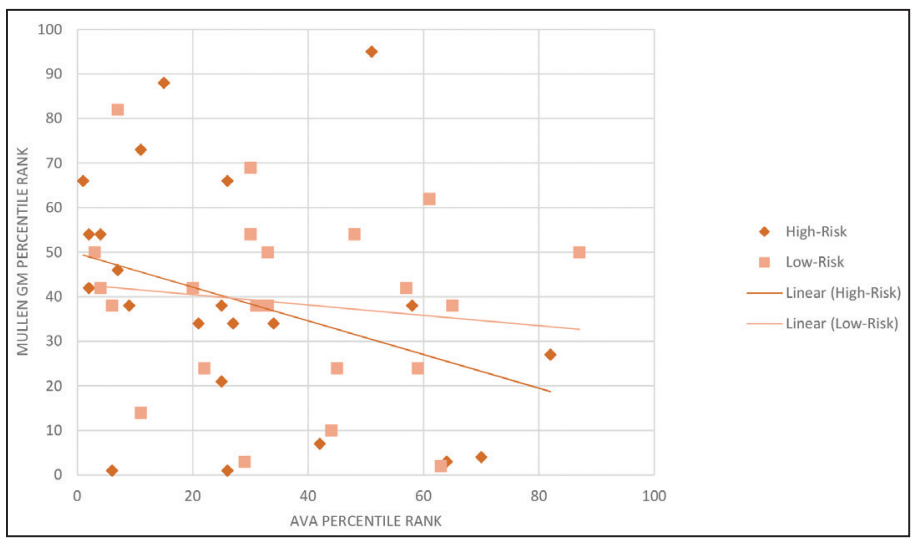

Plot of partial correlations demonstrating no association between Mullen Gross Motor Percentile Rank and the Automatic Vocalization Assessment Percentile Rank (provided by the LENA). The variables shown are just two of the twelve investigated variables. 